\title{
A NOTE ON NEIGHBORHOODS OF ANALYTIC FUNCTIONS HAVING POSITIVE REAL PART
}

\author{
JANICE B. WALKER \\ Department of Mathematics \\ Xavier University \\ Cincinnati, Ohio 45207
}

(Received July 7, 1989 and in revised form October 18, 1989)

ABSTRACT. Let $P$ denote the set of all functions analytic in the unit disk $D=\{z|| z \mid<1\}$ having the form $p(z)=1+\sum_{k=1}^{\infty} p_{k} z^{k}$ with $\operatorname{Re}\{p(z)\}>0$. For $\delta \geqslant 0$, let $N_{\delta}(p)$ be those functions $q(z)=1+\sum_{k=1}^{\infty} q_{k} z^{k^{k=1}}$ analytic in $D$ with $\sum_{k=1}^{\infty}\left|p_{k}-q_{k}\right| \leqslant \delta$. We denote by $P^{\prime}$ the class of functions analytic in $D$ having the form $p(z)=1+\sum_{k=1}^{\infty} p_{k} z^{k}$ with $\left.\operatorname{Re}\{z p(z)]^{\prime}\right\}>0$. We show that $P^{\prime}$ is a subclass of $P$ and determine $\delta$ so that $N_{\delta}(p) \subset P$ for $p \in P^{\prime}$.

KEY WORDS AND PHRASES. Functions having positive real part (Carathéodory class), subordinate function, $\delta$-neighborhood, and convolution (Hadamard product).

1980 AMS SUBJECT CLASSIFICATION CODES. 30C60, $30 \mathrm{C} 99$.

\section{INTRODUCTION}

Let $B$ denote the class of functions $f$ analytic in the unit disk $D=\{z|| z \mid<1\}$ with $f(0)=0$ and $f^{\prime}(0)=1$. For $f(z)=z+\sum_{k=2}^{\infty} a_{k} z^{k}$ in $H$ and $\delta \geqslant 0$, let the $\delta$-neighborhood of $f$ be given by $N_{\delta}(f)=\left\{g(z)=z+\sum_{k=2}^{\infty} b_{k} z^{k}\left|\sum_{k=2}^{\infty} k\right| a_{k}-b_{k} \mid \leqslant \delta\right\}$. For $h(z)=z$, Goodman $[1]$ has shown that $N_{1}(h) \subset S^{*}$ where $S^{*}$ denotes the class of univalent functions in $B$ which are starlike with respect to the origin. St. Ruscheweyh [2] proved that if $f(z)=z+\sum_{k=n+1}^{\infty} a_{k^{2}} z^{k}$ lies in $c$, where $c$ denotes the class of convex univalent functions in $B$, then $N_{\delta}(f) \subset S^{*}$ for $\delta_{n}=2^{-2 / n}$. Fournier [3] found that if C were replaced by

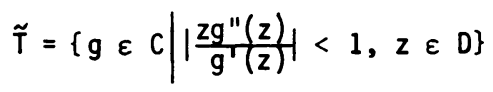

and $S^{*}$ by

$$
T=\left\{g \in S^{\star}|| \frac{z g^{\prime}(z)}{g(z)}-1 \mid<1, z \in D\right\}
$$

then $N_{\delta_{n}}(f) \subset T$ for $\delta_{n}=e^{-1 / n}$. Brown [4] extended the results of St. Ruscheweyh and Fournier and provided simpler proofs. We shall focus on a class of functions directly related to $S^{*}$ and to other classes of univalent functions. Let $P$ denote the class of 
functions analytic in $|z|<1$ having the form $p(z)=1+\sum_{k=1}^{\infty} p_{k} z^{k}$ with $\operatorname{Re}\{p(z)\}>0$ for $|z|<1$. This family is usually called the Carathéodory class. For $f$ in $H$, recall that $f \varepsilon S^{*}$ if and only if $p(z)=z f^{\prime}(z) / f(z)$ lies in $P$.

Let $P^{\prime}$ denote the class of functions analytic in $|z|<1$ having the form $p(z)=1+\sum_{k=1}^{\infty} p_{k} z^{k}$ with $\operatorname{Re}\{[z p(z)] '\}>0$ for $|z|<1$. In this paper we shall define a neighborhood of $p \varepsilon P^{\prime}$ and determine $\delta>0$ so that $N_{\delta}(p) \subset P$.

2. PRELIMINARY RESULTS.

We begin by defining $P$ and $P^{\prime}$ in terms of subordination. Recall that $g$ is subordinate to $h$, written $g \prec h$, if $g(z)=h(w(z))$ where $w$ is analytic in $|z|<1, w(0)=0$ and $|w(z)|<1$ for $|z|<1$. Since $\frac{1+z}{1-z}$ has positive real part in $|z|<1$, is univalent, and is 1 when $z=0$, it is not difficult to show that

$$
p \in P \text { if and only if } p(z) \prec \frac{1+z}{1-z}
$$

and that

$$
p \varepsilon P^{\prime} \text { if and only if }[\mathrm{zp}(z)]^{\prime} \prec \frac{1+z}{1-z} \text {. }
$$

One can also show that $P^{\prime} \subset P$. For according to (2.2), if $p \in P^{\prime}$ then

and thus we have

$$
[z p(z)] ' \prec \frac{1+z}{1-z}
$$

$$
\frac{[z p(z)]^{\prime}}{[z]^{\prime}} \prec \frac{1+z}{1-z} \text {. }
$$

Since $\frac{1+z}{1-z}$ is convex and univalent, we can apply a lemma (see Brown [5], p. 192) to obtain

$$
\frac{z p(z)}{z} \prec \frac{1+z}{1-z}
$$

from which it follows that

$$
p(z) \prec \frac{1+z}{1-z}
$$

Hence, by (2.1) $p \in P$ and $P^{\prime} \subset P$.

Now let us establish a criterion for a given function to belong to $P$. By (2.1) $q \varepsilon P$ if and only if $q(z) \prec \frac{1+z}{1-z}$. Since $\frac{1+z}{1-z}$ is univalent, then $q \varepsilon P$ if and only if $q(z) \neq \frac{1+e^{i \theta}}{1-e^{i \theta}}$, for $0<\theta<2 \pi$ and $|z|<1$. That is,

$$
q \varepsilon P \text { if and only if }\left(1-e^{i \theta}\right) q(z)-\left(1+e^{i \theta}\right) \neq 0 \text {, }
$$

for $0<\theta<2 \pi,|z|<1$.

We can express (2.3) in terms of convolutions. Let $f$ and $g$ be analytic in the unit disk $D$. Recall that if $f(z)=\sum_{k=0}^{\infty} a_{k} z^{k}$ and $g(z)=\sum_{k=0}^{\infty} b_{k} z^{k}$, then the convolution (or Hadamard product) of $f$ and $g$, denoted by $f * g$, is

$$
f * g=\sum_{k=0}^{\infty} a_{k} b_{k} z^{k} .
$$


Thus, $\left(1-e^{i \theta}\right) q(z)-\left(1+e^{i \theta}\right)$ can be written as

$$
\begin{aligned}
& \left(1-e^{i \theta}\right)\left[\frac{1}{1-z} \star q(z)\right]-\left(1+e^{i \theta}\right) * q(z) \\
& =\left(\frac{1-e^{i \theta}}{1-z}-\left(1+e^{i \theta}\right)\right) \star q(z) .
\end{aligned}
$$

Let $h_{\theta}(z)$ be defined by

$$
h_{\theta}(z)=-\frac{1}{2 e^{i \theta}}\left[\frac{1-e^{i \theta}}{1-z}-\left(1+e^{i \theta}\right)\right] .
$$

Then it follows that $h_{\theta}(0)=1$ and for $0<\theta<2 \pi,|z|<1, q \varepsilon P$ if and only if $h_{\theta}(z) * q(z) \neq 0$.

3. THE MAIN RESULT.

We define a $\delta$-neighborhood of $p$ for $p \in P$.

DEFINITION. For any $p(z)=1+\sum_{k=1}^{\infty} p_{k} z^{k}$ in $P$ and $\delta \geqslant 0$, the $\delta$-neighborhood of $p$, denoted by $N_{\delta}(p)$, is

Our main result is the following theorem.

$$
N_{\delta}(p)=\left\{q(z)=1+\sum_{k=1}^{\infty} q_{k} z^{k}\left|\sum_{k=1}^{\infty}\right| p_{k}-a_{k} \mid \leqslant \delta\right\} .
$$

THEOREM. If $\mathrm{p}(\mathrm{z})=1+\sum_{\mathrm{k}=1}^{\infty} \mathrm{p}_{\mathrm{k}} \mathrm{z}^{\mathrm{k}}$ belongs to $P^{\prime}$, then $\mathrm{N}_{\delta}(\mathrm{p}) \subset P$, where $\delta=2 \ln 2-1$ $\simeq .3862944$. This result is sharp.

We need several lemmas.

LEMMA 1. If $p \in P^{\prime}$, then $z\left(p * h_{\theta}\right)$ is univalent for each $0<\theta<2 \pi$.

PROOF. Fix $0<\theta<2 \pi$. Then

$$
\begin{aligned}
{\left[z\left(p \star h_{\theta}\right)\right]^{\prime} } & =\left[\frac{-z}{2 e^{i \theta}}\left(\left(1-e^{i \theta}\right) p(z)-\left(1+e^{i \theta}\right)\right)\right]^{\prime} \\
& =-\frac{1}{2}\left[z p(z)-\frac{1+e^{i \theta}}{1-e^{i \theta}}\right]^{\prime} \frac{1-e^{i \theta}}{e^{i \theta}} \\
& =-\frac{1}{2}\left[(z p(z))^{\prime}-\frac{1+e^{i \theta}}{1-e^{i \theta}}\right]\left(1-e^{i \theta}\right) e^{-i \theta}
\end{aligned}
$$

By definition of $P^{\prime}$, the range of $(z p(z))^{\prime}$ for $|z|<1$ lies in $\operatorname{Re}(z)>0$ and that of $\frac{1+e^{i \theta}}{1-e^{i \theta}}$ lies on the imaginary axis. Thus, we can choose $\alpha$ so that

$$
\operatorname{Re}\left\{e^{i \alpha}\left[z\left(p * h_{\theta}\right)(z)\right] '\right\}>0
$$

for $|z|<1$, namely $\alpha=\arg \left\{-\left(1-e^{i \theta}\right)^{-1} e^{i \theta}\right\}$. By the Noshiro-Warschawski Theorem (Duren [6], p. 47), $z\left(p \star h_{\theta}\right)$ is univalent for each $\theta, 0<\theta<2 \pi$.

LEMMA 2. If $p \in P^{\prime}$, then $\left|\left[z\left(p * h_{\theta}\right)\right]^{\prime}\right|>\frac{1-r}{1+r}$ for $|z|=r<1,0<\theta<2 \pi$.

PROOF. Using expression (3.1) for $\left|z\left(p * h_{\theta}\right)\right|^{r}$, we define $F(w)=e^{-i \theta}\left(1-e^{i \theta}\right)$ $\left(\frac{1+e^{i \theta}}{1-e^{i \theta}}-w\right)$, where $w=\frac{1+r e^{i t}}{1-r e^{i t}}, 0 \leqslant t \leqslant 2 \pi$. Now $F(w)$ may be rewritten as

$$
\left.F(w)=e^{-i \theta_{\{}}\left(1+e^{i \theta}\right)-\left(1-e^{i \theta}\right) w\right\}, 0<\theta<2 \pi \text {. }
$$

Thus,

$$
|F(w)|=|1+w|\left|\frac{1-w}{1+w}+e^{i \theta}\right|
$$


Since $|1+w|=\left|1+\frac{1+r e^{i t}}{1-r e^{i t}}\right|=\left|\frac{2}{1-r e^{i t}}\right| \geqslant \frac{2}{1+r}$, it is clear that

$$
|F(w)| \geqslant 2 \frac{1-r}{1+r} \text {. }
$$

Since $p \in P^{\prime}$ and (3.1)holds, by letting $w=[z p(z)]$ ' we get the desired inequality. That is

$$
\left|\left[z\left(p * h_{\theta}\right)\right] \cdot\right| \geqslant \frac{1-r}{1+r} \text {. }
$$

The lemma is proved.

LEMMA 3. If $p \in P^{\prime}$, then $\left|p * h_{\theta}\right| \geqslant \delta$, where $\delta=\int_{0}^{1} \frac{1-t}{1+t} d t=2 \ln 2-1$.

PROOF. Let $p \in P^{\prime}$. Then by Lemma $1, z\left(p \star h_{\theta}\right)$ is univalent. For fixed $0<r<1$, choose $z_{0}$ with $\left|z_{0}\right|=r$ such that

$$
\min _{|z|=r}\left|z\left(p \star h_{\theta}\right)\right|=\left|z_{0}\left(p \star h_{\theta}\right)\left(z_{0}\right)\right| \text {. }
$$

Since $z\left(p \star h_{\theta}\right)$ is univalent, the preimage $L$ of the line segment from 0 to $z_{0}\left[\left(p \star h_{\theta}\right)\left(z_{0}\right)\right]$ is an arc inside $|z| \leqslant r$. Hence, for $|z| \leqslant r$ we have

$$
\begin{aligned}
& \left|z\left(p * h_{\theta}\right)\right| \geqslant\left|z_{0}\left(p * h_{\theta}\right)\right| \\
& =\int_{L}\left|\left[z\left(p * h_{\theta}\right)\right] \cdot\right||d z| \\
& \geqslant \int_{0}^{r}\left|\left[z\left(p * h_{\theta}\right)\right] \cdot\right||d z| .
\end{aligned}
$$

Accordingly, we apply Lemma 2 to get

$$
\begin{aligned}
\left|\left[p * h_{\theta}\right](z)\right| & \geqslant \frac{1}{r} \int_{0}^{r}\left|\left[z\left(p * h_{\theta}\right)\right] \cdot\right||d z| \\
& \geqslant \frac{1}{r} \int_{0}^{r} \frac{1-t}{1+t} d t \\
& =\frac{2}{r} \ln (1+r)-1 .
\end{aligned}
$$

The function $g(r)=\frac{2}{r} \ln (1+r)-1$ is decreasing for $r>0$ if $g^{\prime}(r)=$ $\frac{-2}{r^{2}} \ln (1+r)+\frac{2}{r(1+r)}<0$. It is not difficult to show that $r-(1+r) \ln (1+r)$ $\leqslant 0$ for $r \geqslant 0$, from which it follows that $g^{\prime}(r)<0$ for $r>0$. Hence

$$
\left|p \star h_{\theta}\right| \geqslant 2 \ln 2-1 \text {. }
$$

This completes the proof of Lemma 3. Now we may prove the theorem.

PROOF (OF THEOREM). Let $p(z)=1+\sum_{k=1}^{\infty} p_{k} z^{k} \varepsilon P^{\prime}$ and let $\delta$ be as in Lemma 3. We want to show that every $q \in N_{\delta}(p)$ belongs to $P$, where $q(z)=1+\sum_{k=1}^{\infty} q_{k} z^{k}$ is an arbitrary but fixed function in $N_{\delta}(p)$. Hence, $\sum_{k=1}^{\infty}\left|p_{k}-q_{k}\right| \leqslant \delta$. Observe that

$$
\left|h_{\theta} * q\right|=\left|\left(h_{\theta} \star p\right)+h_{\theta} *(q-p)\right|
$$




$$
\begin{aligned}
& \geqslant\left|h_{\theta} \star p\right|-\left|h_{\theta} \star(q-p)\right| \\
& \geqslant \delta-\left|\sum_{k=1}^{\infty} \frac{1-e^{i \theta}}{2}\left(q_{k}-p_{k}\right) z^{k}\right| \\
& >\delta-\sum_{k=1}^{\infty}\left|a_{k}-p_{k}\right| \geqslant \delta-\delta=0 .
\end{aligned}
$$

Therefore, $h_{\theta} * q \neq 0$ for $|z|<1$. By (2.4), it follows that $q \in P$. Consequently, $N_{\delta}(p) \subset P$.

Now we prove that the result is sharp. Let $p(z)$ be defined by $(z p(z))^{\prime}=\frac{1+z}{1-z}$. Then $p(z)=-1-\frac{2}{z} \ln (1-z)$. Now let $q(z)=p(z)+\delta z=-1-\frac{2}{z} \ln (1-z)+\delta z$. Clearly, $q \varepsilon N_{\delta}(p)$. However, as $z \rightarrow-1$, then $q(z) \rightarrow-1+2 \ln 2-\delta=q(-1)$.

Therefore, if $\delta>2$ ln $2-1$, then $q(-1)<0$ and consequently Re $q(z)<0$ for $z$ near -1 . This contradicts $\operatorname{Re} q(z)>0$ for $|z|<1$. This completes the proof of the theorem.

\section{REFERENCES}

1. GOODMAN, A. W., Univalent functions and nonanalytic curves, Proc. Amer. Math. Soc. 8 $(1957), 598-601$.

2. ST. RUSCHEWEYH, Neighborhoods of univalent functions, Proc. Amer. Math. Soc. 81 (1981), 521-527.

3. FOURNIER, R., A note on neighborhoods of univalent functions, Proc. Amer. Math. Soc. 87 (1983), 117-120.

4. BROWN, J. E., Some sharp neighborhoods of univalent functions, Trans. Amer. Math. Soc. 287 (1985), 475-482.

5. BROWN, J. E., Quasiconformal extensions for some geometric subclasses of univalent functions, International Journal of Math. and Math. Sciences (1984), 187-195.

6. DUREN, P. L., Univalent functions, Springer-Verlag, New York, 1983.

7. HALLENBECK, D. J. and MaCGREGOR, T. H., Linear problems and convexity techniques in geometric function theory, Pitman Publishing Limited, 1984. 




Advances in

Operations Research

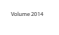

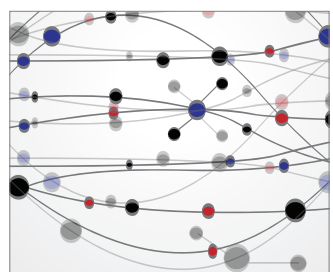

\section{The Scientific} World Journal
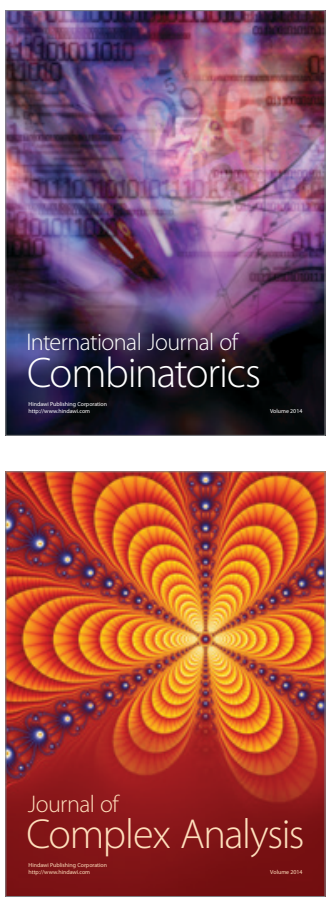

International Journal of

Mathematics and

Mathematical

Sciences
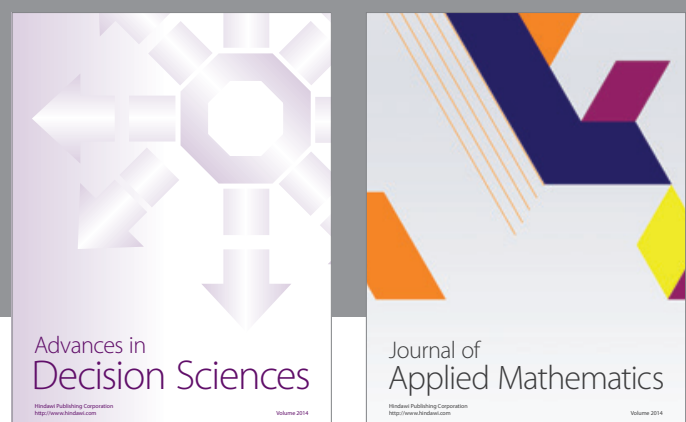

Journal of

Applied Mathematics
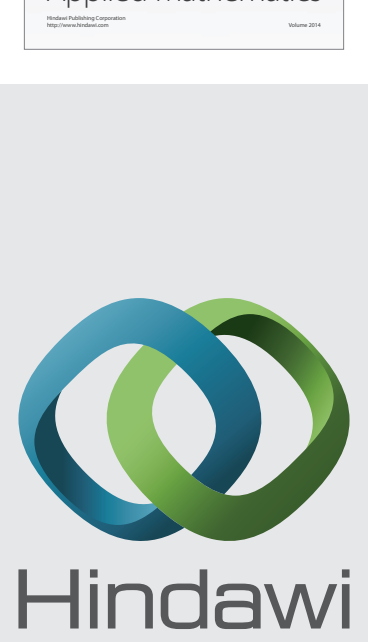

Submit your manuscripts at http://www.hindawi.com


Mathematical Problems in Engineering
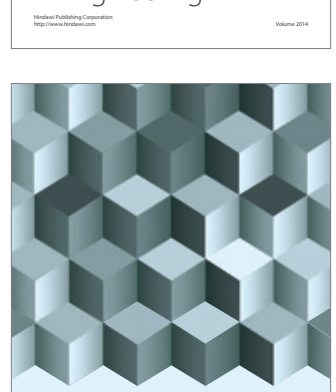

Journal of

Function Spaces
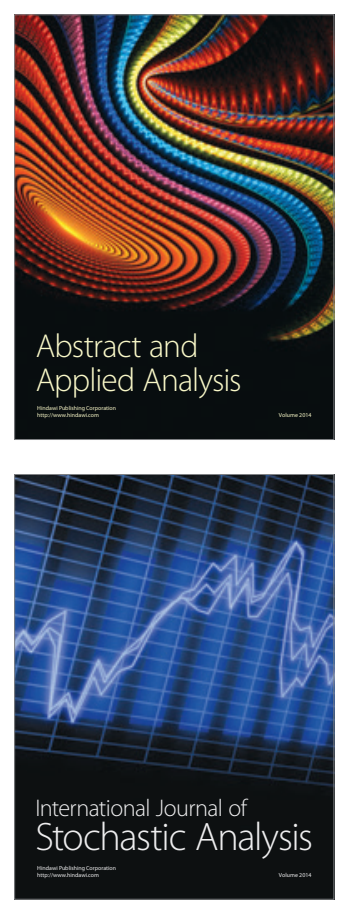

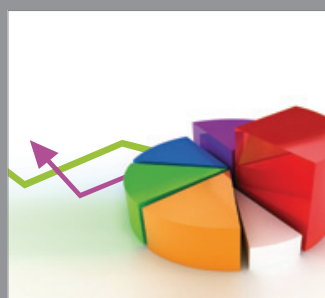

ournal of

Probability and Statistics

Promensencen
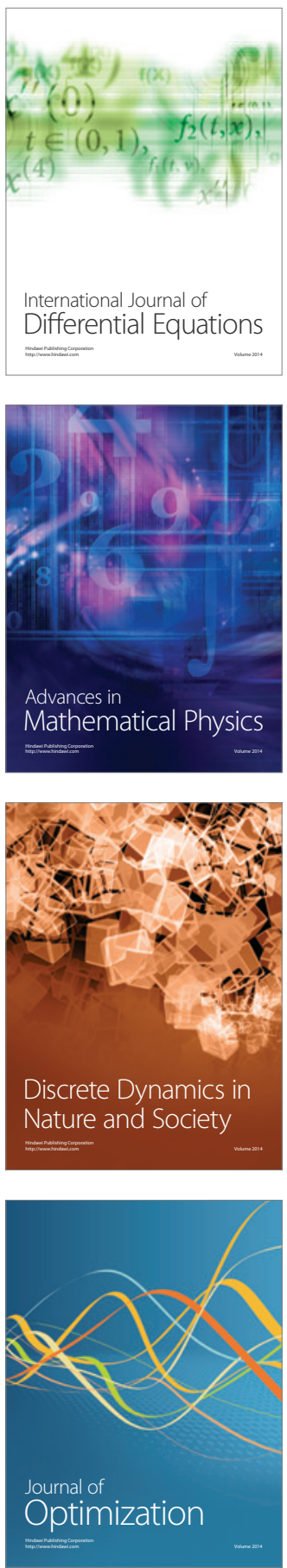